\title{
Familial pneumothorax: towards precision medicine
}

\author{
Rachel M Scott, ${ }^{1}$ Elizabeth P Henske, ${ }^{2}$ Benjamin Raby, ${ }^{3}$ Philip M Boone, ${ }^{4}$ \\ Rosemary A Rusk, ${ }^{5}$ Stefan J Marciniak ${ }^{1,6}$
}

${ }^{1}$ Wellcome Trust/MRC

Cambridge Institute for Medical Research (CIMR), University of

Cambridge, Cambridge, UK ${ }^{2}$ Division of Pulmonary and Critical Care Medicine, Brigham and Women's Hospital, Harvard Medical School, Boston,

Massachusetts, USA

${ }^{3}$ Channing Division of Network Medicine, Division of Pulmonary and Critical Care Medicine,

Pulmonary Genetics Center, Brigham and Women's Hospital, Harvard Medical School, Boston, Massachusetts, USA

${ }^{4}$ Harvard Genetics Training Program, Boston, Massachusetts, USA

${ }^{5}$ Department of Cardiology, Papworth Hospital, Cambridge, UK

${ }^{6}$ Division of Respiratory Medicine, Addenbrooke's Hospital, Cambridge University Hospitals NHS Foundation Trust, Cambridge, UK

\section{Correspondence to} Prof Stefan J Marciniak, Cambridge Institute for Medical Research (CIMR), University of Cambridge, Cambridge CB2 OXY,UK; sjm20@cam.ac.uk

Received 16 October 2017 Revised 29 November 2017 Accepted 4 December 2017 Published Online First 28 December 2017

Check for updates

To cite: Scott RM, Henske EP, Raby $\mathrm{B}$, et al. Thorax 2018:73:270-276

\section{ABSTRACT}

One in 10 patients suffering from primary spontaneous pneumothoraces has a family history of the disorder. Such familial pneumothoraces can occur in isolation, but can also be the presentation of serious genetic disorders with life-threatening vascular or cancerous complications. As the pneumothorax frequently precedes the more dangerous complications by many years, it provides an opportunity to intervene in a focused manner, permitting the practice of precision medicine. In this review, we will discuss the clinical manifestations and underlying biology of the genetic causes of familial pneumothorax.

\section{INTRODUCTION}

In elephants, fusion of the visceral and parietal pleura fixes the lung to the chest wall. ${ }^{1}$ This developmental pleurodesis may have evolved to prevent pneumothoraces caused by the high transmural pressures experienced during trunk-snorkelling. ${ }^{1}$ Humans, by contrast, do not share this adaptation, and so a potential pleural space persists that can fill with air if the lung or chest wall is punctured. A spontaneous pneumothorax occurs when air enters the pleural space in the absence of trauma. Owing to positive transmural pressure across the visceral pleura, this deflates the lung to a degree dependent on how efficiently the puncture closes. ${ }^{2}$ Many spontaneous pneumothoraces occur secondarily to lung pathology, in particular COPD, but those occurring in apparently healthy individuals are labelled 'primary spontaneous pneumothoraces'.

Classically, primary spontaneous pneumothoraces present in patients between the ages of 18 and 40 , and are fourfold more common in men, with an annual incidence of 4-18 per 100000 in men and 1.2-6 per 100000 in women. ${ }^{3}$ Affected individuals are often tall and thin, perhaps reflecting the higher transmural pressures experienced at the apices of elongated lungs, although it is equally possible that taller individuals have connective tissues of lower tensile strengths. The pathogenic mechanisms of primary spontaneous pneumothorax remain incompletely understood, but thoracic CT identifies ipsilateral apical subpleural blebs or bullae in $89 \%$ of patients with primary spontaneous pneumothorax compared with $20 \%$ of controls. ${ }^{4}$ Likewise, at surgery, between $76 \%$ and $100 \%$ are found to have blebs or bullae, although this may be biased towards more severe cases. ${ }^{25}$

A family history of pneumothorax can be elicited in $10 \%$ of individuals presenting with spontaneous pneumothorax. ${ }^{6}$ Indeed, the term familial spontaneous pneumothorax was first coined a century ago in the report of an affected pair of identical twins. ${ }^{7}$ The diagnosis of familial cases is important to optimise current management and to allow more serious complications of the causative mutation to be anticipated and treated (figure 1). It is therefore important to recognise these cases and elucidate the molecular defect causing a familial pneumothorax, enabling proactive, individualised care-a true application of the so-called 'precision medicine'.

Inherited connective tissue disorders account for a proportion of such cases. A second group of familial pneumothoraces is defined by mutations that affect tumour suppressor pathways. ${ }^{8-10}$ These mutations frequently lead to the formation of pulmonary cysts that rupture to cause pneumothorax. Again, early diagnosis is essential to put in place the necessary surveillance and management of life-threatening extrathoracic complications. ${ }^{11}$ The health benefits can be extended by identifying relatives who share the mutation and are also at risk of disease. A proportion of individuals with familial pneumothorax currently fail to be given a genetic diagnosis, but the advent of inexpensive genome sequencing offers an opportunity to define the genetic landscape of familial pneumothorax.

\section{DEFECTS OF TUMOUR SUPPRESSORS}

A group of disorders that can present as familial pneumothorax involves mutations in tumour suppressor genes. Two classic examples are BirtHogg-Dubé (BHD) syndrome and tuberous sclerosis complex (TSC), both of which have been linked to the kinase mechanistic target of rapamycin (mTOR), a master regulator of protein synthesis that links environmental cues with cell growth and proliferation. ${ }^{12}$ Activation of mTOR complex 1 (mTORC1) enhances protein translation, inhibits autophagy and initiates a series of metabolic adaptations to promote cell growth, including increases in nucleotide biosynthesis. mTORC1 is tonically inhibited by hamartin (TSC1), tuberin (TSC2) and TBC1D $7 .{ }^{13}$ In turn, inhibition of these TSC regulators by growth signals or mutations can activate (or disinhibit) the mTOR pathway, leading to complex multisystem phenotypes in patients with TSC (discussed below) and to sporadic human malignancies, renal cystic disease and many other disorders.

\section{BHD syndrome}

BHD syndrome is the most common genetic cause of familial pneumothorax, accounting for 10\%-15\% of all cases. ${ }^{14}{ }^{15}$ It is an autosomal dominant condition caused by inactivating mutations in the FLCN gene that encodes the folliculin protein. ${ }^{16-18}$ Patients with BHD can develop pulmonary cysts, facial fibrofolliculomas, renal cell carcinoma and renal oncocytoma. Loss of folliculin enhances cell proliferation, ${ }^{19}$ but the mechanisms of this are unclear. 


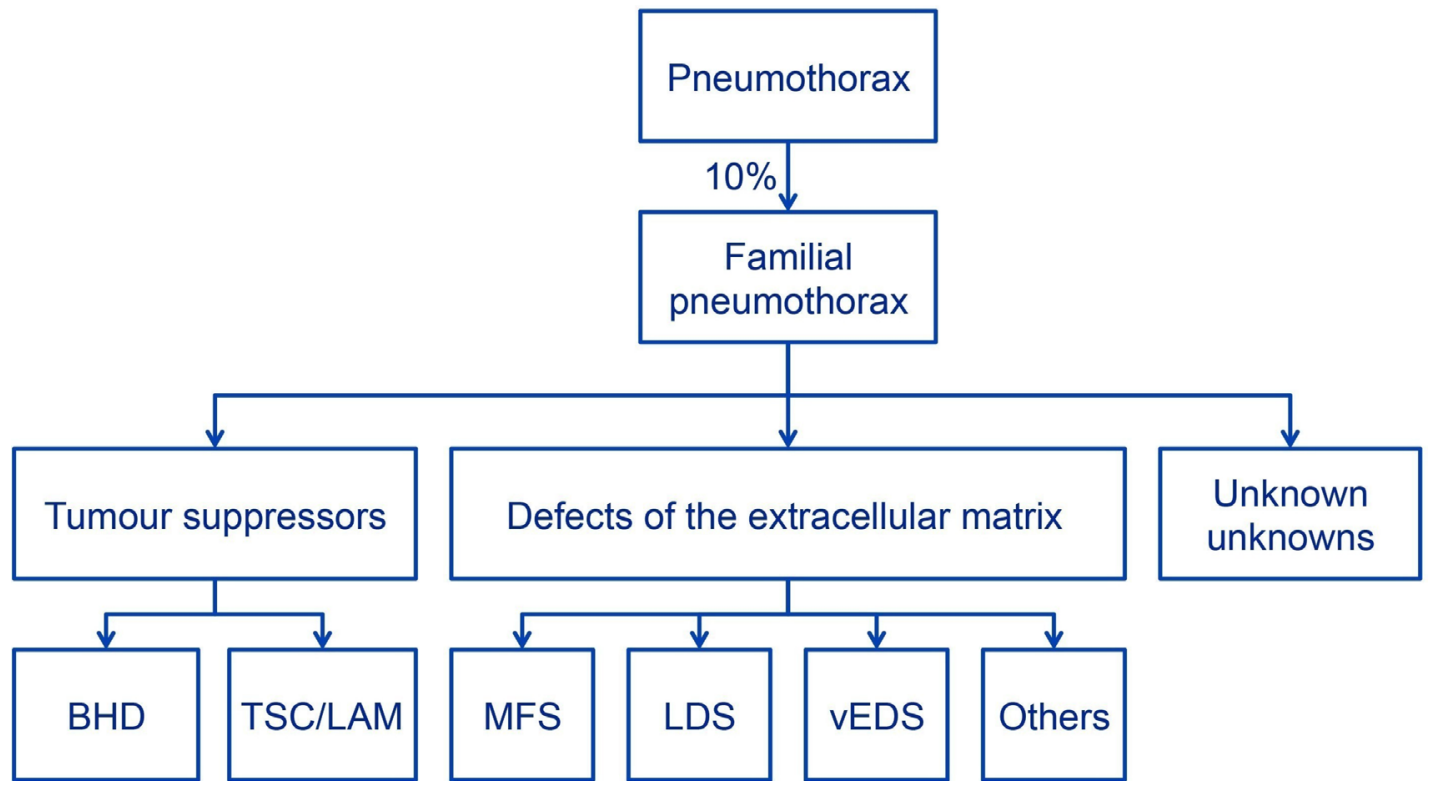

Figure 1 Familial pneumothorax. Familial pneumothorax accounts for $10 \%$ of all primary spontaneous pneumothoraces. These can be further subdivided between those caused by mutations of tumour suppressors and those caused by defects of the extracellular matrix. Approximately half of all familial pneumothoraces currently remain unclassifiable by standard clinical and genetic testing (unknown unknowns). BHD, Birt-Hogg-Dubé syndrome; LAM, lymphangioleiomyomatosis; LDS, Loeys-Dietz syndrome; MFS, Marfan syndrome; TSC, tuberous sclerosis complex; vEDS, vascular Ehlers-Danlos syndrome.

Folliculin has a complex connection to the mTOR pathway. In mice, inactivating Flcn mutations have been found to both enhance and inhibit mTORC1 activity. ${ }^{20-22}$ In cells, folliculin is required for activation of $\mathrm{mTORC} 1$ at the lysosome, with folliculin-deficient cells showing decreased mTORC1 activity. ${ }^{23} 24$

Diagnosis of BHD syndrome can be challenging. Fibrofolliculomas are the most common skin lesion in this syndrome and appear as small dome-shaped papules on the face, neck and upper trunk from the third decade onwards. These skin lesions can aid in diagnosis, but the dermatological features are absent in a quarter of adults with FLCN mutations, and so the disorder can often present instead with pneumothorax or renal cancer. ${ }^{25}$ Renal malignancies occur later in life and are curable surgically if identified when still small. ${ }^{26}$ For this reason, a diagnosis of BHD following a pneumothorax provides individuals and their affected relatives with the benefits of annual surveillance imaging, ideally with renal MRI scanning, to identify early renal tumours when they can be easily treated.

Lung cysts and spontaneous pneumothorax are common features of BHD (figure 2). Histologically, cysts are lined with epithelium and can have internal septations, but the parenchyma between them is usually normal. ${ }^{27}{ }^{28} \mathrm{Up}$ to $86 \%$ of patients with FLCN mutations have lung cysts, which on thoracic CT imaging have a characteristic predominantly basal distribution and irregular shape. ${ }^{29}{ }^{30}$ Forty per cent of cysts abut the pleura, which likely explains why patients with BHD have a 24\%-48\% lifetime risk of pneumothorax. ${ }^{3132}$ In contrast to the general population, individuals with FLCN mutations do not show an obvious difference in the incidence of pneumothorax between the sexes nor is there a detectable interaction with smoking. ${ }^{32}$ In this condition, spontaneous pneumothorax is most likely to develop in the fourth and fifth decades - the median age of first pneumothorax being 38 years. The presence and size of cysts both correlate significantly with the likelihood of developing pneumothorax. ${ }^{16}$

Remarkably, although the clinical evidence points towards a causal relationship between cyst formation and the development of pneumothorax, the mechanistic link between FLCN mutation and cyst development is not known. In a series of patients with heterozygous FLCN mutations, cyst tissue consistently stained positively for phosphorylate S6 ribosomal protein, a classical mTORC1 target, suggesting activation of mTOR. ${ }^{27}$ This led to a suggestion that cysts might represent hamartomatous

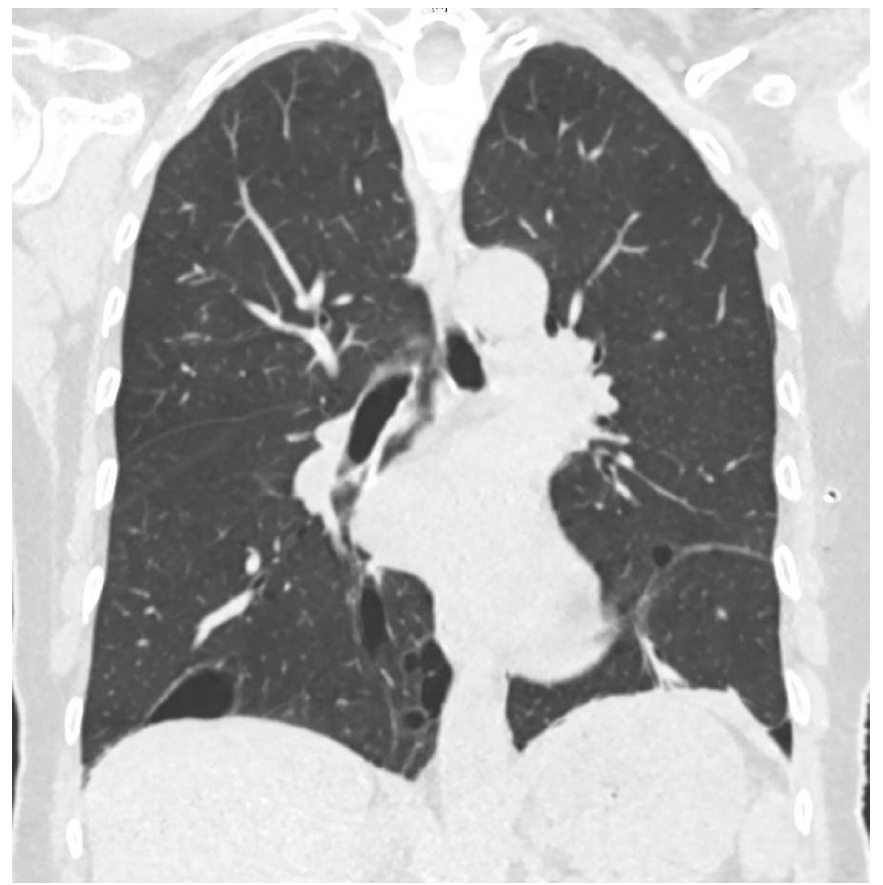

Figure 2 Birt-Hogg-Dubé syndrome. Coronal reconstruction of CT of a patient with Birt-Hogg-Dubé syndrome who had undergone previous left-sided pleurectomy. Note the basal and medial preponderance of irregular cysts, some of which are multiseptated. Reproduced with permission from Nikolić and Marciniak. ${ }^{101}$ 
alveolus formation. Indeed, human alveolar cells from patients with FLCN mutations show evidence of mTORC1 activation. ${ }^{33}$ However, folliculin can also bind to plakophilin-4, a protein involved in the formation of adherens junctions, ${ }^{3435}$ and cell-cell adhesion is enhanced when cells lack either folliculin or plakophilin-4. ${ }^{333536}$ This led to the proposal of a 'stretch hypothesis' in which enhanced cell-cell adhesion results from reduced formation of the folliculin-plakophilin-4 complex. ${ }^{36}$ Consequently, reduced expression of folliculin would diminish the compliance of lung parenchyma, rendering it more susceptible to alveolar rupture during cycles of inspiration and expiration. ${ }^{35-37}$ Since most tidal respiration occurs in the lower zones of the lung, the stretch hypothesis offers an appealing mechanism to explain the distribution of lung cysts in BHD. Nevertheless, the mTOR and stretch hypotheses are not mutually exclusive.

Determining the true nature of the disease mechanism(s) is more than an academic concern. It is necessary to enable the development of rational therapies. At present, apart from surveillance for renal tumours, specific therapies for BHD are lacking. Antagonism of mTOR has been proposed as a potential strategy because in Flcn knockout mice rapamycin has been shown to reduce the development of renal cysts and extend survival. ${ }^{22} 38$ However, given the uncertainty (discussed above) about whether folliculin is an activator or an inhibitor of mTORC1, and the uncertainty about whether renal cysts in the Flcn knockout mice are an appropriate surrogate for pneumothorax and/or renal cell carcinoma in patients with $\mathrm{BHD}$, it will be important to determine the impact of mTORC1 inhibition in the human disease. Currently, a phase II trial of everolimus, an mTORC1 allosteric inhibitor, is ongoing for BHD syndrome-associated renal cancer (NCT02504892).

From a clinical perspective, establishing a correct diagnosis of BHD enables early detection of renal cell carcinomas in both the proband and in undiagnosed family members who also carry the FLCN mutation. All patients with BHD should have regular renal imaging, typically every 2 years if no tumours are detected or more often to monitor the growth of small tumours.

\section{Tuberous sclerosis and lymphangioleiomyomatosis}

TSC is caused by inactivating mutations in the TSC1 and TSC2 genes. $^{39}$ These encode tumour suppressors, and so loss of heterozygosity or inactivation of the wild-type allele, which almost always occurs via somatic mutational events, can lead to hamartoma development in a variety of organs, typically the brain, kidney, skin, retina and lung. ${ }^{40}$ The relevance of tuberous sclerosis to pneumothorax stems from its strong association with lymphangioleiomyomatosis (LAM). Sporadic LAM is rare, affecting only 1 in 1000000 women of childbearing age, and appears to be caused in most cases by somatic mutations in the TSC2 gene. $^{4142}$ By contrast, tuberous sclerosis occurs in 1 in 5000-10 000 births, ${ }^{43}$ and CT screening studies suggest that $34 \%-80 \%$ of women with TSC mutations (and 0\%-10\% of men) have pulmonary cysts consistent with LAM. ${ }^{44-46}$

The female preponderance of LAM is hypothesised to reflect a sensitivity to female sex hormones since disease progression can accelerate dramatically during pregnancy and the disease progresses more rapidly during the premenopausal years. Both LAM and angiomyolipoma cells stain positively for oestrogen and/or progesterone receptors. ${ }^{47}$ The nature of the germline TSC mutation does not appear to influence the risk of developing LAM, although in general patients with TSC1 mutations develop disease of less severity when compared with patients with TSC2 mutations. $^{48}$ LAM carries a 60\%-81\% lifetime risk

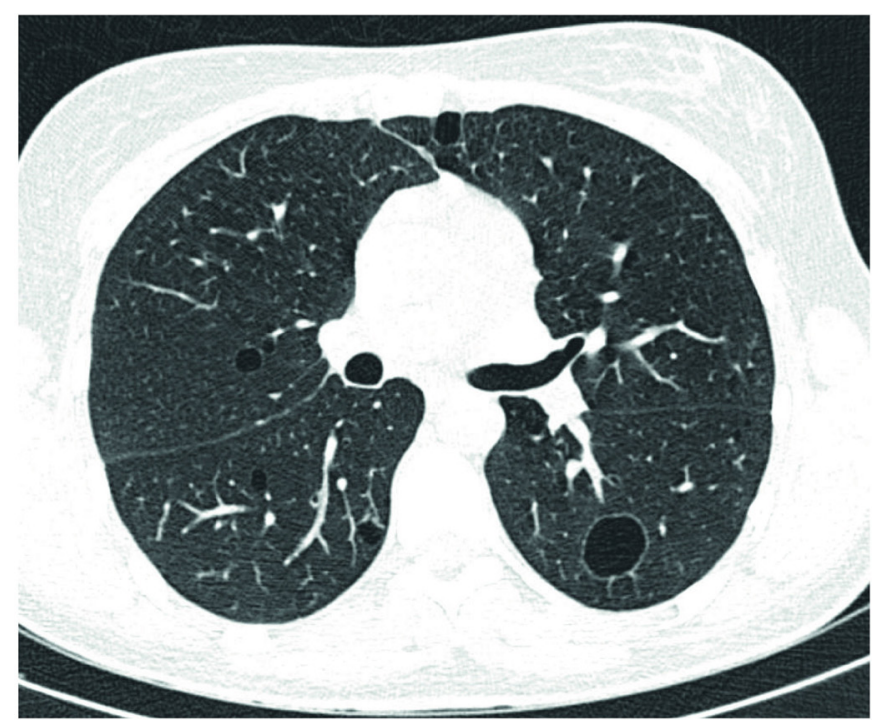

Figure 3 Lymphangioleiomyomatosis. Axial reconstruction of CT of a patient with lymphangioleiomyomatosis. Note the multiple, bilateral thin-walled cysts of varying sizes, some with a subpleural location.

of pneumothorax, and recurrence rates are more than $70 \% .^{9} 10$ Unlike BHD, the thin-walled pulmonary cysts in LAM distribute evenly throughout all regions of the lung ${ }^{49-51}$ (figure 3 ). Histologically LAM is characterised by an abnormal proliferation of smooth muscle-like cells in the alveolar septa that cause progressive cystic lung destruction. The so-called 'LAM cells' characteristically stain with antibody HMB-45, which recognises gp100, a melanocyte marker, leading some to suggest they arise from a melanocyte and/or neural crest lineage precursor. ${ }^{52}$

Some patients with LAM suffer progressive loss of lung function that can eventually necessitate lung transplantation. Circulating VEGF-D is a validated diagnostic biomarker of LAM and may also have utility in predicting the likelihood of disease progression. ${ }^{53}$ Importantly, in those patients with progressive loss of lung function, inhibition of mTORC1 has proven successful in delaying the progression of cystic lung disease and preserving lung function, although the loss of lung function recurs when the drug is stopped, so lifelong therapy appears to be necessary. ${ }^{1154}$

Distinguishing between TSC-LAM (with germline TSC1 or TSC2 gene mutations) and sporadic LAM can be challenging. All women with LAM should be screened for renal angiomyolipomas, which occur in both TSC-LAM and sporadic LAM and can spontaneously haemorrhage. Women with apparently sporadic LAM who are considering having children should be carefully screened for clinical evidence of TSC (imaging of the brain, MRI or CT imaging of the kidneys, and formal dermatological exam for manifestations of TSC) because of the autosomal dominant transmission of germline TSC gene mutations. Some women with apparently sporadic LAM who lack germline TSC gene mutations may have a somatic mosaic form of TSC. Additional considerations for women with LAM considering pregnancy, related to the possible effects of pregnancy-related hormonal changes on the progression of LAM, should be handled in an individualised basis.

\section{TRUE CONNECTIVE TISSUE DISORDERS}

The biomechanical properties that determine a tissue's propensity for spontaneous rupture are dependent on the extracellular matrix secreted by component cells. Mutations that affect the mechanical properties of matrix proteins, such as those 


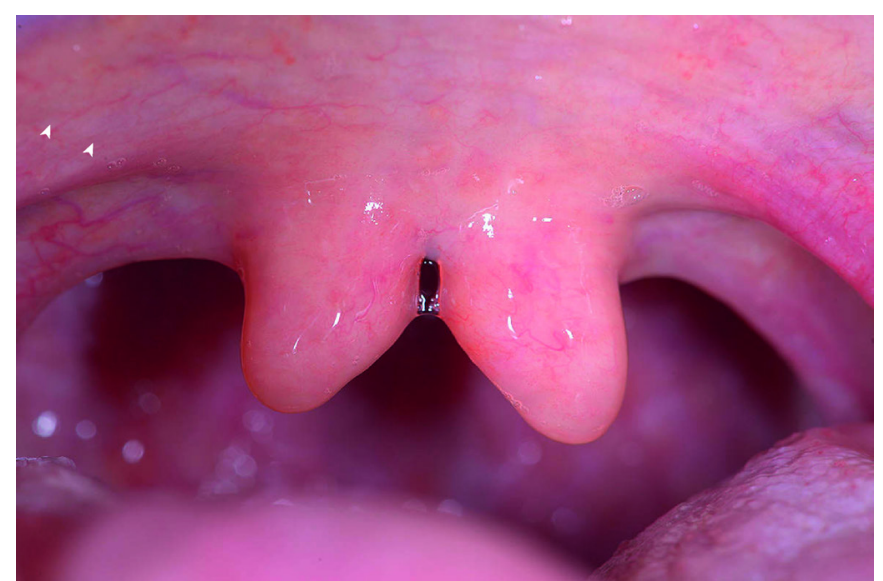

Figure 4 Loeys-Dietz syndrome. Photograph of bifid uvula characteristic of Loeys-Dietz syndrome. ${ }^{69}$

implicated in the connective tissue disorders, therefore have direct effects on the tensile strength and behaviour of many tissues. This is particularly important to hollow viscera that experience significant positive transmural pressures, such as blood vessels and the lungs. Consequently, the same mutation that causes the visceral pleura to fail, causing a pneumothorax, can sometimes cause life-threatening arterial rupture. Identification of those patients at risk of vascular catastrophe enables pre-emptive pharmacological treatment, monitoring and, when required, surgical reinforcement or replacement of affected vessels. In this context, even a small, non-threatening pneumothorax represents a sentinel event, serving as a harbinger for potentially lethal vascular complications.

\section{Marfan syndrome}

Spontaneous pneumothorax occurs in 4.4\%-10.5\% of individuals with Marfan syndrome, which represents a 1000-fold excess compared with the general population. ${ }^{55}$ Indeed, pneumothorax is one of the phenotypes that contribute to the systemic scores used to evaluate patients with the syndrome (see https://www. marfan.org/dx/score). Marfan syndrome is transmitted in an autosomal dominant manner due to mutation of the FBN1 gene. FBN1 is highly polymorphic and prone to mutation. As a result, $25 \%$ of cases result from de novo mutations, ${ }^{56}$ and thus can present in the absence of a family history. FBN1 encodes a glycoprotein, fibrillin-1, which forms microfibrils closely associated with elastin fibres in tendons, skin, cartilage, periosteum and the cardiovascular system. Pathogenic mutations therefore have widespread manifestations. Abnormal bone growth leads to tall stature, disproportionately long limbs and digits (arachnodactyly), scoliosis, pectus excavatum or carinatum, and pes planus, while dysfunction of the suspensory ligaments of the lens can cause subluxation of the lens. In the cardiovascular system, aortic dilatation, particularly of the root, is widely recognised. Other cardiac features include, for example, valve prolapse, most commonly of the mitral valve with associated regurgitation, enlargement of the proximal pulmonary artery and cardiomyopathy.

The mechanism of pneumothorax in Marfan syndrome might be explained either as a consequence of the physiological impact of the body habitus of affected individuals, through increased focal parenchymal tension by musculoskeletal deformity, faulty connective tissue integrity from lack of fibrillin or altered elastin, or through biochemical effects via aberrant transforming growth factor $\beta$ (TGF $\beta$ ) signalling. As mentioned above, elongation of the thorax can lead to increased apical transmural pressures across the visceral pleura and so contribute to bleb formation. The frequency of blebs in patients with Marfan syndrome is $5 \%-9.6 \%, 55$ and those who develop blebs are 10 -fold more likely to develop pneumothorax than those without, $25 \%$ vs $2.7 \%{ }^{55}$ However, in many patients with Marfan syndrome, the extreme height is due to increased extremity length without an increase in torso height. Frequently, patients have significant musculoskeletal deformity, including kyphoscoliosis or pectus deformities. These are occasionally severe and could contribute to local parenchymal tension. ${ }^{57} 58$

In addition to its structure functions, fibrillin- 1 also has a role in regulating TGF $\beta$ signalling, which may be relevant to the pathology of Marfan syndrome. Mice deficient in Fbn1 have abnormally elevated TGF $\beta$ signalling with accompanying distal airspace enlargement and aortic dilatation, which are antecedents of spontaneous pneumothorax and aortic rupture. ${ }^{5960}$ Mechanistic support for the importance of this function on fibrillin-1 comes from experiments in which administration of TGF $\beta$-neutralising antibody attenuated these manifestations. Angiotensin receptor blockade can also antagonise TGF $\beta$ signalling, which may account for the protection afforded by losartan, but not $\beta$-adrenergic receptor blockade, against aortic dilatation in Fbn1 knockout mice. ${ }^{59}$ A small study found angiotensin II receptor blockade slowed the rate of aortic root dilatation in children with Marfan syndrome, ${ }^{61}$ which led to the initiation of several randomised control trials, many of which have yet to report their findings. One study, COMPARE, has reported a beneficial effect of losartan in reducing aortic root dilatation in adults compared with placebo, ${ }^{62}$ although other studies have detected no benefit of losartan over $\beta$-blockade. ${ }^{6364}$ In a recent head-to-head comparison, there were no significant differences in the progression of ascending aorta dilatation between atenolol-treated versus losartan-treated groups over 3 years. ${ }^{65}$ Interestingly, a subanalysis of the COMPARE study suggested that the nature of the FBN1 mutation might dictate a patient's response to angiotensin II receptor blockade. ${ }^{66}$ Studies have not evaluated whether angiotensin II receptor blockade improves pneumothorax risk in patients with Marfan syndrome.

\section{Loeys-Dietz syndrome}

Loeys-Dietz syndrome was first described as a multisystem disorder caused by defects of TGF $\beta$ receptor function leading to features including midline fusion defects (bifid uvula, hypertelorism, cleft palate), widespread arterial involvement with vascular tortuosity and high risk of aneurysms and dissection, along with skeletal defects (scoliosis and elongation of the limbs) ${ }^{67}$ (figure 4). Although the original description of this autosomal dominant condition did not include pulmonary features, considering the phenotypic similarities between Loeys-Dietz syndrome and Marfan syndrome, it is unsurprising that LoeysDietz syndrome has now been linked with the development of spontaneous pneumothorax. ${ }^{68} 69$ In the American National Registry of Genetically Triggered Thoracic Aortic Aneurysms and Cardiovascular Conditions (GenTAC), 4 of 73 patients with Loeys-Dietz syndrome developed pneumothorax. ${ }^{70}$ More recently, pneumothorax has been recognised as a presenting feature of the syndrome. ${ }^{69}$

There are currently five subtypes of Loeys-Dietz syndrome, classified by their mutations in components of the TGF $\beta$ signalling pathway: TGFBR1, TGFBR2, SMAD3, TGFB2 and TGFB3. ${ }^{71}$ Paradoxically, loss-of-function mutations in these genes result in 
elevated TGF $\beta$ signalling that, as with Marfan syndrome, may underlie the pathology of Loeys-Dietz syndrome. ${ }^{72}$ TGF $\beta \beta$ regulates many processes including angiogenesis and wound healing via cell surface receptors that signal both via a canonical pathway involving phosphorylation of SMAD transcription factors and via non-canonical SMAD-independent pathways. Dysregulation of TGF $\beta$ signalling results in structural defects of the extracellular matrix perhaps due to an imbalance between deposition of the extracellular matrix, mediated by the canonical pathway, and matrix degradation, regulated by the non-canonical pathway. Components of the extracellular matrix can also feedback on TGF $\beta$ signalling because microfibrils within the matrix contain TGF $\beta$-binding proteins, such as fibrillin-1, that can sequester TGF $\beta .^{73}$ This could account for the increased TGF $\beta$ signalling seen when fibrillin-1 is lost in models of Marfan syndrome. ${ }^{59}$

\section{Ehlers-Danlos syndrome}

Ehlers-Danlos syndrome (EDS) comprises a group of connective tissue disorders characterised by joint hypermobility, hyperextensible skin, easy bruising and dystrophic scarring, which are caused by mutations in a variety of genes. ${ }^{74}$ Most varieties of EDS have no recognised connection with lung disease, but vascular EDS (vEDS, also known as EDS IV) is a rare cause of spontaneous pneumothorax. Although vEDS is uncommon with a prevalence of less than 1 in $100000,{ }^{75}$ pneumothorax can precede other life-threatening complications by several years, thus enabling recognition at an earlier stage. The majority of patients with vEDS suffer a major complication such as spontaneous rupture of arteries (particularly middle-sized arteries), intestines or gravid uterus before the age of 40 years. vEDS is caused by autosomal dominant mutations in the COL3A1 gene, which encodes the collagen type III alpha 1 chain. ${ }^{76}$ These mutations account for the phenotype because type III collagen is necessary for the integrity of both the pulmonary blood vasculature and the lung parenchyma. ${ }^{77}$ Consequently, defects in COL3A1 predispose to pulmonary haemorrhage and the formation of parenchymal cysts or cavities. ${ }^{77-79}$ Data from the GenTAC registry indicate that pneumothoraces occur in as many as $15 \%(16 / 107)$ of individuals with vEDS. Although the link between vEDS and spontaneous pneumothorax has been known for many years, low levels of clinical suspicion result in a majority of the published cases being diagnosed only following lung biopsy. ${ }^{79-84}$ This is partially explained by the tendency of vEDS to resemble a pulmonary vasculitic process both clinically and on cross-sectional imaging, although autoimmune screens are typically negative. ${ }^{85}$

The shared pulmonary and vascular features of vEDS and other true connective tissue disorders suggest a degree of common pathology. Indeed, individuals with vEDS have increased circulating levels of TGF $\beta 1$ and TGF $\beta 2$ and their dermal fibroblasts secrete more TGF 32 than controls. ${ }^{86}$ However, the effects of therapies targeting TGF $\beta$ signalling have yet to be studied in this condition. The only human clinical trial to date in vEDS examined the effect of celiprolol, a mixed $\beta_{1}$-adrenoceptor antagonist and $\beta_{2}$-adrenoceptor agonist. ${ }^{87}$ This identified a moderate protective effect in reducing the occurrence of arterial rupture or dissection from $50 \%$ to $20 \%$.

\section{The rarest of the rare}

Homocystinuria and cutis laxa are rare even among true connective tissue disorders, with estimated incidences of 1 per $4000 \quad 000$ and 1 per $60000-100000$, respectively. ${ }^{88-97}$ Homocystinuria is an autosomal recessive disorder caused by the deficiency of cystathionine- $\beta$ - synthase, resulting in increased plasma levels of homocysteine and methionine. Homocysteinylation of proteins, including fibrillin-1, manifests in many ways, for example osteoporosis, downwards subluxation of the lens and neuropsychiatric disease. Case studies suggest that pneumothoraces also occur, ${ }^{88-97}$ but vascular events (thromboses and strokes) account for the majority of the condition's morbidity, with half of patients affected before the age of $30 .^{88-97}$ Although homocystinuria is screened for by the neonatal heel prick test, a false- negative rate of $0.03 \%$ means that diagnosis may not occur until complications present. ${ }^{8-97}$ Also, patients may have been born prior to the introduction of routine testing for homocystinuria or in countries that still do not test. Importantly, homocystinuria can be treated through dietary modification and so early diagnosis can yield large benefits. ${ }^{88-97}$ Individuals with cutis laxa have abnormal elastic fibres that result in hypoelastic skin and sometimes in the development of emphysema. Again, case reports suggest that spontaneous pne umothorax is also a manifestation of the disease. ${ }^{88-97}$ Mutations in several genes can cause cutis laxa, including ELN, FBLN4 and FBLN5, encoding elastin and fibrin proteins of the extracellular matrix. In mice, expression of a hypomorphic allele of Fbn4 was shown to cause aneurysm formation and to be associated with increased TGF $\beta$ signalling, suggesting that treatments developed for the more common true connective disease might prove useful for these patients.$^{88-97}$

\section{$\boldsymbol{\alpha}_{1}$-ANTITRYPSIN DEFICIENCY}

Pneumothorax can complicate emphysema caused by excessive degradation of the extracellular matrix. Since $1 \%-2 \%$ of cases of COPD are due to $\alpha_{1}$-antitrypsin deficiency, pneumothorax can be the presenting feature of mutations in the SERPINA1 gene. $^{98}{ }^{99}$ While the benefits of replacement therapies remain debatable, early diagnosis permits beneficial lifestyle modification such as smoking cessation. Many of the disease-associated mutations cause polymerisation of the protein within hepatocytes to cause liver dysfunction through effects on endoplasmic reticulum structure and luminal protein mobility. ${ }^{98} 100$ Indeed, $13 \%$ of patients homozygous for the $\mathrm{Z}$ allele die of liver disease. Diagnosis therefore permits prospective monitoring for hepatic complications, which include cirrhosis and hepatocellular carcinoma.

\section{CONCLUDING REMARKS}

Familial pneumothorax rather than being a single entity comprises a number of genetic disorders. These can have severe extrapulmonary manifestations ranging from renal cancer to aortic rupture. Since pneumothorax is a common early presentation of these life-shortening diseases, it offers an important opportunity to make a diagnosis that can facilitate precision medicine. Family tracing can subsequently identify many at-risk mutation carriers and so the health benefits are potentially widereaching. The diagnoses themselves are frequently straightforward when clinical context and radiology are considered, with genetic tests being confirmatory. However, a high level of clinical suspicion is necessary if diagnoses are to be made and, above all, a family history must be sought proactively.

Despite the large heritable component in spontaneous pneumothorax, up to half of cases remain unclassifiable following thorough investigation. For this reason, familial pneumothorax has recently been added by Genomics England to the list of pulmonary disorders within the 100000 Genomes Project (Rare Disease Conditions Eligibility Criteria V.1.6.0 (2016), Genomics England). The criteria for recruitment are (1) primary 
spontaneous pneumothorax, (2) one or more affected relative and (3) prior testing for FLCN or FBN1 mutations if suggested by the clinical and radiological findings. It is hoped that this systematic genome-wide analysis of familial pneumothorax will explain the aetiology of many of the remaining cases, be they formes frustes of known disorders or entirely new genetic entities. Eventually, targeted gene panel testing will enter routine clinical practice for the investigation of pneumothorax.

Contributors SJM and RMS were responsible for conception and overall design of the review. All authors contributed substantially to drafting the article.

Funding The work was supported by funds from the British Lung Foundation, Medical Research Council, and Addenbrooke's and Papworth hospitals, and from funds from the Precision Medicine Initiative, Department of Pathology, Brigham and Women's Hospital, Boston.

Competing interests None declared.

Provenance and peer review Not commissioned; externally peer reviewed.

(c) Article author(s) (or their employer(s) unless otherwise stated in the text of the article) 2018. All rights reserved. No commercial use is permitted unless otherwise expressly granted.

\section{REFERENCES}

1 West JB. Snorkel breathing in the elephant explains the unique anatomy of its pleura. Respir Physiol 2001;126:1-8.

2 Sahn SA, Heffner JE. Spontaneous pneumothorax. N Eng/ J Med 2000;342:868-74.

3 Melton LJ, Hepper NG, Offord KP. Incidence of spontaneous pneumothorax in Olmsted County, Minnesota: 1950 to 1974. Am Rev Respir Dis 1979;120:1379-82.

4 Mitlehner W, Friedrich M, Dissmann W. Value of computer tomography in the detection of bullae and blebs in patients with primary spontaneous pneumothorax. Respiration 1992:59:221-7.

5 Schramel FM, Sutedja TG, Janssen JP, et al. Prognostic factors in patients with spontaneous pneumothorax treated with video-assisted thoracoscopy. Diagn Ther Endosc 1995;2:1-5

6 Abolnik IZ, Lossos IS, Zlotogora J, et al. On the inheritance of primary spontaneous pneumothorax. Am J Med Genet 1991;40:155-8.

7 Faber E. Spontaneous pneumothorax in two siblings. Hospitalstid 1921;64:573-4.

8 Hopkins TG, Maher ER, Reid E, et al. Recurrent pneumothorax. Lancet 2011;377:1624.

9 Johnson SR, Cordier JF, Lazor R, et al. European Respiratory Society guidelines for the diagnosis and management of lymphangioleiomyomatosis. Eur Respir $J$ 2010;35:14-26.

10 Steagall WK, Glasgow CG, Hathaway OM, et al. Genetic and morphologic determinants of pneumothorax in lymphangioleiomyomatosis. Am J Physiol Lung Cell Mol Physiol 2007;293:L800-8.

11 McCormack FX, Inoue Y, Moss J, et al. Efficacy and safety of sirolimus in lymphangioleiomyomatosis. N Eng/ J Med 2011;364:1595-606.

12 Shimobayashi M, Hall MN. Making new contacts: the mTOR network in metabolism and signalling crosstalk. Nat Rev Mol Cell Biol 2014;15:155-62.

13 Saxton RA, Sabatini DM. mTOR signaling in growth, metabolism, and disease. Cell 2017;168:960-76.

14 Graham RB, Nolasco M, Peterlin B, et al. Nonsense mutations in folliculin presenting as isolated familial spontaneous pneumothorax in adults. Am J Respir Crit Care Med 2005; 172:39-44

15 Ebana H, Mizobuchi T, Kurihara M, et al. Novel clinical scoring system to identify patients with pneumothorax with suspicion for Birt-Hogg-Dubé syndrome. Respirology 2017. doi: 10.1111/resp.13191 [Epub ahead of print 27 Sep 2017].

16 Toro JR, Pautler SE, Stewart L, et al. Lung cysts, spontaneous pneumothorax, and genetic associations in 89 families with Birt-Hogg-Dubé syndrome. Am J Respir Crit Care Med 2007;175:1044-53.

17 Toro JR, Wei MH, Glenn GM, et al. BHD mutations, clinical and molecular genetic investigations of Birt-Hogg-Dubé syndrome: a new series of 50 families and a review of published reports. J Med Genet 2008;45:321-31.

18 Nickerson ML, Warren MB, Toro JR, et al. Mutations in a novel gene lead to kidney tumors, lung wall defects, and benign tumors of the hair follicle in patients with the Birt-Hogg-Dubé syndrome. Cancer Cell 2002;2:157-64.

19 Hasumi H, Hasumi Y, Baba M, et al. H255Y and K508R missense mutations in tumour suppressor folliculin (FLCN) promote kidney cell proliferation. Hum Mol Genet 2017;26:354-66

20 Hartman TR, Nicolas E, Klein-Szanto A, et al. The role of the Birt-Hogg-Dubé protein in mTOR activation and renal tumorigenesis. Oncogene 2009;28:1594-604.

21 Furuya M, Nakatani Y. Birt-Hogg-Dube syndrome: clinicopathological features of the lung. J Clin Pathol 2013;66:178-86.
22 Baba M, Furihata M, Hong SB, et al. Kidney-targeted Birt-Hogg-Dube gene inactivation in a mouse model: Erk1/2 and Akt-mTOR activation, cell hyperproliferation, and polycystic kidneys. J Nat/ Cancer Inst 2008;100:140-54.

23 Tsun ZY, Bar-Peled L, Chantranupong L, et al. The folliculin tumor suppressor is GAP for the RagC/D GTPases that signal amino acid levels to mTORC1. Mol Cell 2013:52:495-505.

24 Petit CS, Roczniak-Ferguson A, Ferguson SM. Recruitment of folliculin to lysosomes supports the amino acid-dependent activation of Rag GTPases. J Cell Biol 2013;202:1107-22.

25 Menko FH, van Steensel MA, Giraud S, et al. Birt-Hogg-Dubé syndrome: diagnosis and management. Lancet Oncol 2009;10:1199-206.

26 Pavlovich CP, Grubb RL, Hurley K, et al. Evaluation and management of renal tumors in the Birt-Hogg-Dubé syndrome. J Urol 2005;173:1482-6.

27 Furuya M, Tanaka R, Koga S, et al. Pulmonary cysts of Birt-Hogg-Dubé syndrome: a clinicopathologic and immunohistochemical study of 9 families. Am J Surg Pathol 2012;36:589-600.

28 Koga S, Furuya M, Takahashi Y, et al. Lung cysts in Birt-Hogg-Dubé syndrome: histopathological characteristics and aberrant sequence repeats. Pathol Int 2009:59:720-8

29 Agarwal PP, Gross BH, Holloway BJ, et al. Thoracic CT findings in birt-hogg-dube syndrome. AJR Am J Roentgenol 2011;196:349-52.

30 Zbar B, Alvord WG, Glenn G, et al. Risk of renal and colonic neoplasms and spontaneous pneumothorax in the Birt-Hogg-Dubé syndrome. Cancer Epidemiol Biomarkers Prev 2002:11:393-400.

31 Rehman HU. Birt-Hogg-Dubé syndrome: report of a new mutation. Can Respir J 2012:19:193-5.

32 Houweling AC, Gijezen LM, Jonker MA, et al. Renal cancer and pneumothorax risk in Birt-Hogg-Dubé syndrome; an analysis of 115 FLCN mutation carriers from 35 BHD families. Br J Cancer 2011;105:1912-9.

33 Khabibullin D, Medvetz DA, Pinilla M, et al. Folliculin regulates cell-cell adhesion, AMPK, and mTORC1 in a cell-type-specific manner in lung-derived cells. Physiol Rep 2014:2:e12107

34 Nahorski MS, Seabra L, Straatman-Iwanowska A, et al. Folliculin interacts with p0071 (plakophilin-4) and deficiency is associated with disordered RhoA signalling, epithelial polarization and cytokinesis. Hum Mol Genet 2012:21:5268-79.

35 Medvetz DA, Khabibullin D, Hariharan V, et al. Folliculin, the product of the birthogg-dube tumor suppressor gene, interacts with the adherens junction protein p0071 to regulate cell-cell adhesion. PLoS One 2012;7:e47842.

36 Kennedy JC, Khabibullin D, Henske EP. Mechanisms of pulmonary cyst pathogenesis in Birt-Hogg-Dube syndrome: The stretch hypothesis. Semin Cell Dev Biol 2016:52:47-52

37 Johannesma PC, Houweling AC, van Waesberghe JH, et al. The pathogenesis of pneumothorax in birt-hogg-dubé syndrome: a hypothesis. Respirology 2014:19:1248-50.

38 Chen J, Futami K, Petillo D, et al. Deficiency of FLCN in mouse kidney led to development of polycystic kidneys and renal neoplasia. PLoS One 2008;3:e3581.

39 Curatolo P, Bombardieri R, Jozwiak S. Tuberous sclerosis. Lancet 2008;372:657-68.

40 Giannikou K, Malinowska IA, Pugh TJ, et al. Whole exome sequencing identifies TSC1/TSC2 biallelic loss as the primary and sufficient driver event for renal angiomyolipoma development. PLoS Genet 2016;12:e1006242.

41 Carsillo T, Astrinidis A, Henske EP. Mutations in the tuberous sclerosis complex gene TSC2 are a cause of sporadic pulmonary lymphangioleiomyomatosis. Proc Nat/ Acad Sci U S A 2000;97:6085-90.

42 Fujita A, Ando K, Kobayashi E, et al. Detection of low-prevalence somatic TSC2 mutations in sporadic pulmonary lymphangioleiomyomatosis tissues by deep sequencing. Hum Genet 2016;135:61-8.

43 von Ranke FM, Zanetti G, e Silva JL, et al. Tuberous sclerosis complex: state-of-theart review with a focus on pulmonary involvement. Lung 2015;193:619-27.

44 Muzykewicz DA, Sharma A, Muse V, et al. TSC1 and TSC2 mutations in patients with lymphangioleiomyomatosis and tuberous sclerosis complex. J Med Genet 2009:46:465-8

45 Moss J, Avila NA, Barnes PM, et al. Prevalence and clinical characteristics of lymphangioleiomyomatosis (LAM) in patients with tuberous sclerosis complex. Am J Respir Crit Care Med 2001:164:669-71.

46 Cudzilo CJ, Szczesniak RD, Brody AS, et al. Lymphangioleiomyomatosis screening in women with tuberous sclerosis. Chest 2013:144:578-85.

47 Logginidou H, Ao X, Russo I, et al. Frequent estrogen and progesterone receptor immunoreactivity in renal angiomyolipomas from women with pulmonary lymphangioleiomyomatosis. Chest 2000;117:25-30.

48 Strizheva GD, Carsillo T, Kruger WD, et al. The spectrum of mutations in TSC1 and TSC2 in women with tuberous sclerosis and lymphangiomyomatosis. Am J Respir Crit Care Med 2001:163:253-8

49 Avila NA, Chen CC, Chu SC, et al. Pulmonary lymphangioleiomyomatosis: correlation of ventilation-perfusion scintigraphy, chest radiography, and CT with pulmonary function tests. Radiology 2000;214:441-6.

50 Chorianopoulos D, Stratakos G. Lymphangioleiomyomatosis and tuberous sclerosis complex. Lung 2008:186:197-207.

51 Koo HK, Yoo CG. Multiple cystic lung disease. Tuberc Respir Dis 2013;74:97-103. 
52 Zhe $X$, Schuger L. Combined smooth muscle and melanocytic differentiation in lymphangioleiomyomatosis. J Histochem Cytochem 2004;52:1537-42.

53 Young LR, Inoue Y, McCormack FX. Diagnostic potential of serum VEGF-D for lymphangioleiomyomatosis. N Engl J Med 2008;358:199-200.

54 Yao J, Taveira-DaSilva AM, Jones AM, et al. Sustained effects of sirolimus on lung function and cystic lung lesions in lymphangioleiomyomatosis. Am J Respir Crit Care Med 2014;190:1273-82.

55 Karpman C, Aughenbaugh GL, Ryu JH. Pneumothorax and bullae in Marfan syndrome. Respiration 2011;82:219-24.

56 Dietz HC. In: Pagon RA, Adam MP, Ardinger HH, eds. Marfan syndrome. Seattle, WA: GeneReviews(R), 1993.

57 Streeten EA, Murphy EA, Pyeritz RE. Pulmonary function in the Marfan syndrome. Chest 1987;91:408-12.

58 Saita K, Murakawa T, Kawano H, et al. Chest wall deformity found in patients with primary spontaneous pneumothorax. Asian Cardiovasc Thorac Ann 2013;21:582-7.

59 Habashi JP, Judge DP, Holm TM, et al. Losartan, an AT1 antagonist, prevents aortic aneurysm in a mouse model of Marfan syndrome. Science 2006;312:117-21.

60 Neptune ER, Frischmeyer PA, Arking DE, et al. Dysregulation of TGF-beta activation contributes to pathogenesis in Marfan syndrome. Nat Genet 2003:33:407-11.

61 Brooke BS, Habashi JP, Judge DP, et al. Angiotensin II blockade and aortic-root dilation in Marfan's syndrome. N Engl J Med 2008;358:2787-95.

62 Groenink M, den Hartog AW, Franken R, et al. Losartan reduces aortic dilatation rate in adults with Marfan syndrome: a randomized controlled trial. Eur Heart J 2013:34:3491-500.

63 Lacro RV, Dietz HC, Sleeper LA, et al. Atenolol versus losartan in children and young adults with Marfan's syndrome. N Engl J Med 2014;371:2061-71.

64 Milleron O, Arnoult F, Ropers J, et al. Marfan Sartan: a randomized, double-blind, placebo-controlled trial. Eur Heart J 2015:36:2160-6.

65 Forteza A, Evangelista A, Sánchez V, et al. Efficacy of losartan vs. atenolol for the prevention of aortic dilation in Marfan syndrome: a randomized clinical trial. Eur Heart J 2016;37:978-85.

66 Franken R, den Hartog AW, Radonic T, et al. Beneficial outcome of losartan therapy depends on type of FBN1 mutation in Marfan syndrome. Circ Cardiovasc Genet 2015;8:383-8.

67 Loeys BL, Chen J, Neptune ER, et al. A syndrome of altered cardiovascular, craniofacial, neurocognitive and skeletal development caused by mutations in TGFBR1 or TGFBR2. Nat Genet 2005;37:275-81.

68 MacCarrick G, Black JH, Bowdin S, et al. Loeys-Dietz syndrome: a primer for diagnosis and management. Genet Med 2014;16:576-87.

69 Chambers JE, Dalton LE, Subramanian DN, et al. Spontaneous pneumothorax can be associated with TGFBR2 mutation. Eur Respir J 2015;46:1832-5.

70 Habashi JP, Oswald GL, Holmes KW, et al. Prevalence and predictors of pneumothorax in patients with connective tissue disorders enrolled in the GenTAC (National registry of genetically triggered thoracic aortic aneurysms and cardiovascular conditions) Registry. 2013. American Society of Human Genetics Meeting http://www.ashg.org/2013meeting/abstracts/fulltext/f130122591.htm.

71 Bertoli-Avella AM, Gillis E, Morisaki H, et al. Mutations in a TGF- $\beta$ ligand, TGFB3, cause syndromic aortic aneurysms and dissections. J Am Coll Cardiol 2015;65:1324-36.

72 Van Laer L, Dietz H, Loeys B, et al. Loeys-Dietz syndrome. Adv Exp Med Biol 2014;802:95-105.

73 Unsöld C, Hyytiäinen M, Bruckner-Tuderman L, et al. Latent TGF-beta binding protein LTBP-1 contains three potential extracellular matrix interacting domains. J Cell Sci 2001;114(Pt 1):187-97.

74 Beighton P, De Paepe A, Steinmann B, et al. Ehlers-Danlos syndromes: revised nosology, Villefranche, 1997. Ehlers-Danlos National Foundation (USA) and EhlersDanlos Support Group (UK). Am J Med Genet 1998;77:31-7.

75 Chuman H, Trobe JD, Petty EM, et al. Spontaneous direct carotid-cavernous fistula in Ehlers-Danlos syndrome type IV: two case reports and a review of the literature. $J$ Neuroophthalmol 2002;22:75-81.

76 Tsipouras P, Byers PH, Schwartz RC, et al. Ehlers-Danlos syndrome type IV: cosegregation of the phenotype to a COL3A1 allele of type III procollagen. Hum Genet 1986;74:41-6.
77 Watanabe A, Kawabata Y, Okada O, et al. Ehlers-Danlos syndrome type IV with few extrathoracic findings: a newly recognized point mutation in the COL3A1 gene. Eur Respir J 2002;19:195-8.

78 Ishiguro T, Takayanagi N, Kawabata Y, et al. Ehlers-Danlos syndrome with recurrent spontaneous pneumothoraces and cavitary lesion on chest $X$-ray as the initial complications. Intern Med 2009;48:717-22.

79 Abrahamsen BJ, Kulseth MA, Paus B. A 19-year-old man with relapsing bilateral pneumothorax, hemoptysis, and intrapulmonary cavitary lesions diagnosed with vascular Ehlers-Danlos syndrome and a novel missense mutation in COL3A1. Chest 2015;147:e166-e170.

80 Dar RA, Wani SH, Mushtaque M, et al. Spontaneous hemo-pneumothorax in a patient with Ehlers-Danlos syndrome. Gen Thorac Cardiovasc Surg 2012;60:587-9.

81 Nakagawa H, Wada H, Hajiro T, et al. Ehlers-Danlos Syndrome Type IV with bilateral pneumothorax. Intern Med 2015;54:3181-4.

82 Kadota Y, Fukui E, Kitahara N, et al. Total pleural covering technique for intractable pneumothorax in patient with Ehlers-Danlos syndrome. Gen Thorac Cardiovasc Surg 2016;64:425-8.

83 Geake JB, Ritchey DM, Burke J, et al. Sudden death in a young male with a recent pneumothorax: a case report. Eur Respir Rev 2014;23:145-7.

84 Savasta S, Leoni MC, Strocchio L, et al. A rare cause of recurrent spontaneous pneumothorax. Clin Pediatr 2011;50:456-8.

85 Badawi RA, Brent LH, Feinstein DE. Mimics of vasculitis: vascular Ehlers-Danlos syndrome masquerading as polyarteritis nodosa. J Rheumatol 2009;36:1845-6.

86 Morissette R, Schoenhoff F, Xu Z, et al. Transforming growth factor- $\beta$ and inflammation in vascular (type IV) Ehlers-Danlos syndrome. Circ Cardiovasc Genet 2014;7:80-8.

87 Ong KT, Perdu J, De Backer J, et al. Effect of celiprolol on prevention of cardiovascular events in vascular Ehlers-Danlos syndrome: a prospective randomised, open, blindedendpoints trial. Lancet 2010;376:1476-84.

88 Van Maldergem L, Loeys B. In: Pagon RA, Adam MP, Ardinger HH, Wallace SE, Amemiya A, eds. FBLN5-Related Cutis Laxa. Seattle (WA): GeneReviews(R), 1993.

89 Walter JH, Jahnke N, Remmington T. Newborn screening for homocystinuria. Cochrane Database Syst Rev 2015;10:CD008840.

90 Cochran FB, Packman S. Homocystinuria presenting as sagittal sinus thrombosis. Eur Neurol 1992;32:1-3.

91 Hatch TP. Utilization of exogenous thymidine by Chlamydia psittaci growing in the thymidine kinase-containing and thymidine kinase-deficient $L$ cells. J Bacteriol 1976;125:706-12.

92 Yap S. Classical homocystinuria: vascular risk and its prevention. J Inherit Metab Dis 2003:26:259-65.

93 Peterschmitt MJ, Simmons JR, Levy HL. Reduction of false negative results in screening of newborns for homocystinuria. N Engl J Med 1999;341:1572-6.

94 Kumar T, Sharma GS, Singh LR. Homocystinuria: therapeutic approach. Clin Chim Acta 2016:458:55-62.

95 Genevieve D, Baumann C, Huber C, et al. A novel form of syndromic cutis laxa with facial dysmorphism, cleft palate, and mental retardation. J Med Genet 2004;41:e77.

96 Nascimento GM, Nunes CS, Menegotto PF, et al. Cutis laxa: case report. An Bras Dermatol 2010;85:684-6.

97 Hanada K, Vermeij M, Garinis GA, et al. Perturbations of vascular homeostasis and aortic valve abnormalities in fibulin-4 deficient mice. Circ Res 2007;100:738-46.

98 Greene CM, Marciniak SJ, Teckman J, et al. $\alpha 1$-Antitrypsin deficiency. Nat Rev Dis Primers 2016:2:16051.

99 Lin YC, Chiu WK, Chang H, et al. Spontaneous pneumothorax in flight as first manifestation of alpha-1 antitrypsin deficiency. Aviat Space Environ Med 2008;79:704-6.

100 Dickens JA, Ordóñez A, Chambers JE, et al. The endoplasmic reticulum remains functionally connected by vesicular transport after its fragmentation in cells expressing Z- $\alpha 1$-antitrypsin. Faseb J 2016;30:4083-97.

101 Nikolić MZ, Marciniak SJ. Familial pneumothorax. Respiratory disease in practice 2017:25:8-11. 